\title{
Molecular mechanism of Forkhead box M1 inhibition by thiostrepton in breast cancer cells
}

\author{
MESAYAMAS KONGSEMA ${ }^{1-3}$, SUDTIRAK WONGKHIEO ${ }^{1}$, MATTAKA KHONGKOW ${ }^{4}$, ERIC W.-F. LAM ${ }^{5}$, \\ PHANSIRI BOONNOY ${ }^{2,3,6}$, WANWIPA VONGSANGNAK ${ }^{1,2}$ and JIRASAK WONG-EKKABUT ${ }^{2,3,6,7}$
}

\author{
${ }^{1}$ Department of Zoology, and ${ }^{2}$ Computational Biomodelling Laboratory for Agricultural Science and Technology (CBLAST), \\ Faculty of Science, Kasetsart University, Bangkok 10900; ${ }^{3}$ Thailand Center of Excellence in Physics (ThEP Center), \\ Commission on Higher Education, Bangkok 10400; ${ }^{4}$ National Nanotechnology Centre (NANOTEC), \\ National Science and Technology Development Agency, Pathum Thani 12120, Thailand; ${ }^{5}$ Department of \\ Surgery and Cancer, Imperial College London, Hammersmith Hospital Campus, London W12 0NN, UK; \\ ${ }^{6}$ Department of Physics, and ${ }^{7}$ Specialized Center of Rubber and Polymer Materials for Agriculture and Industry (RPM), \\ Faculty of Science, Kasetsart University, Bangkok 10900, Thailand
}

Received January 15, 2019; Accepted July 3, 2019

DOI: $10.3892 / o r .2019 .7225$

\begin{abstract}
Breast cancer is the most common type of malignancies in women worldwide, and genotoxic chemotherapeutic drugs are effective by causing DNA damage in cancer cells. However, $>90 \%$ of patients with metastatic cancer are resistant to chemotherapy. The Forkhead box M1 (FOXM1) transcription factor plays a pivotal role in the resistance of breast

Correspondence to: Dr Mesayamas Kongsema, Department of Zoology, Faculty of Science, Kasetsart University, Bangkok 10900, Thailand

E-mail: mesayamas.k@ku.ac.th

Dr Jirasak Wong-Ekkabut, Computational Biomodelling Laboratory for Agricultural Science and Technology (CBLAST), Faculty of Science, Kasetsart University, Bangkok 10900, Thailand

E-mail: jirasak.w@ku.ac.th
\end{abstract}

Abbreviations: ATB, Automated force field Topology Builder; ChIP-seq, chromatin immunoprecipitation sequencing; DBD, DNA binding domain; DMEM, Dulbecco's modified Eagle's medium; DMSO, dimethyl sulfoxide; FBS, foetal bovine serum; FKH, Forkhead box domain; FOXM1, Forkhead box M1; ITC, isothermal titration calorimetry; MD, molecular dynamics; MMP-9, matrix metallopeptidase 9; MTT, 3-(4, 5-dimethylthiazolyl-2)-2, 5-diphenyltetrazoliumbromide; NMR, nuclear magnetic resonance; PME, Particle-mesh Ewald; RMSF, root-mean-square fluctuation; RT-PCR, reverse transcription polymerase chain reaction; SA- $\beta$-gal, senescence-associated $\beta$-galactosidase; siRNA, small interfering RNA; SPC, simple point-charge model; TAD, transactivation domain; V-rescale, velocity-rescale; VMD, visual molecular dynamics; X-gal, 5-bromo-4-chloro-3-indolyl- $\beta$-d-galactopyrano side; XIAP, X-linked inhibitor of apoptosis protein

Key words: Forkhead box M1, molecular dynamics simulations, thiostrepton, senescence, breast cancer cancer cells to chemotherapy by promoting DNA damage repair following genotoxic drug treatment. The aim of the present study was to investigate the inhibition of the FOXM1 protein by thiostrepton, a natural antibiotic produced by the Streptomyces species. Experimental studies were designed to examine the effectiveness of thiostrepton in downregulating FOXM1 mRNA expression and activity, leading to senescence and apoptosis of breast cancer cells. The cytotoxicity of thiostrepton in breast cancer was determined using cell viability assay. Additionally, thiostrepton treatment decreased the mRNA expression of cyclin B1 (CCNB1), a downstream target of FOXM1. The present results indicated that thiostrepton inhibited FOXM1 mRNA expression and its effect on $C C N B 1$. Molecular dynamic simulations were performed to study the interactions between FOXM1-DNA and thiostrepton after molecular docking. The results revealed that the possible mechanism underlying the inhibitory effect of thiostrepton on FOXM1 function was by forming a tight complex with the DNA and FOXM1 via its binding domain. Collectively, these results indicated that thiostrepton is a specific and direct inhibitor of the FOXM1 protein in breast cancer. The findings of the present study may lead to the development of novel therapeutic strategies for breast cancer and help overcome resistance to conventional chemotherapeutic drugs.

\section{Introduction}

Chemotherapy has been demonstrated to reduce the risk of metastasis by killing the cancer cells circulating in the bloodstream, thereby increasing the disease-free survival rates (1). The common types of chemotherapy for cancer treatment are DNA intercalating agents, such as epirubicin and doxorubicin, causing lethal DNA damage in the cells (2). Although chemotherapy is highly potent in killing cancer cells, some patients do not respond to chemotherapy, and are referred to as chemoresistant. There is clear evidence that the Forkhead box M1 (FOXM1) transcription factor is a protein crucial for 
DNA damage repair and plays a key role in cancer progression and chemotherapy resistance (3-6). It was previously demonstrated $(5,7,8)$ that doxorubicin-resistant breast cancer cells exhibit a significant increase in FOXM1 protein expression levels, with no FOXM1 degradation after doxorubicin treatment (3). The active forms of FOXM1, particularly the FOXM1b and FOXM1c isoforms, can increase DNA double-strand repair via homologous recombination $(3,5)$. Conversely, inhibition of FOXM1 expression and activity has been revealed to result in a marked decrease in cancer metastatic rates and an increase in the sensitivity of cancer cells to chemotherapy $(4,6,9)$. Targeting FOXM1 in cancer cells may prove to be a novel approach to cancer therapy and may also enhance the efficacy of combination therapies by increasing chemosensitivity in cancer patients.

Recent research has demonstrated that thiostrepton can specifically inhibit FOXM1 activity, leading to proliferation arrest and apoptosis $(10,11)$. Thiostrepton is a natural antibiotic product, containing a thiazole ring, that is produced by the Steptomyces species and is recognized to have a broad spectrum of antibacterial and antiparasitic properties (10). Notably, Kwok et al (11) demonstrated that thiostrepton can inhibit cell proliferation by causing G1/S and G2/M cell cycle arrest. Additionally, thiostrepton has been shown to decrease the expression of $\mathrm{X}$-linked inhibitor of apoptosis protein and matrix metallopeptidase 9 in FOXM1-overexpressing cells (12).

Cellular senescence is an anticancer event caused by irreversible cell cycle arrest $(13,14)$. Our previous research has demonstrated that knockdown of FOXM1 by small interfering RNA (siRNA) may cause DNA damage-induced senescence (4), namely irreversible cell cycle arrest $(13,14)$. However, the mechanism through which thiostrepton inhibits FOXM1 activity is unclear, since the interaction between thiostrepton and FOXM1 at the molecular level remains elusive $(15,16)$. Gartel $(15,17,18)$ indicated that thiostrepton does not directly bind to and form complexes with FOXM1; instead, it stabilizes the negative regulators (p21 $1^{\mathrm{Cipl}}$ and $\mathrm{p} 53$ ) of FOXM1 by inhibiting the proteasome degradation pathway, similar to other proteasome inhibitors, such as siomycin A and MG132 $(15,19,20)$. By contrast, our previous study demonstrated that FOXM1 protein levels were increased when MCF-7 breast cancer cells were treated with MG132 (8). In addition, other experimental and computational results demonstrated that thiostrepton can interact directly with the DNA-binding domain (DBD) of FOXM1 $(16,21)$. Isothermal titration calorimetry (ITC) measurement of the thiostrepton-FOXM1 interaction also revealed that thiostrepton binds directly to FOXM1 with 1:1 stoichiometry (16). Moreover, the binding of thiostrepton and FOXM1 was also confirmed by affinity pull-down assays (16). However, crucial evidence remains elusive due to limitations in time and length scale of the binding process $(15,17,18,22)$. The advances in computational simulations offer an alternative complementary approach, and high-throughput screenings for small molecules binding to the dimer FOXM1-DNA complex have been successfully carried out (21).

The aim of the present study was to investigate the role of thiostrepton in inducing senescence in cancer cells, in order to gain a better understanding of its antiproliferative properties and its functional doses at low concentrations. The associa- tion between the effects of thiostrepton on FOXM1 inhibition and cellular senescence was also investigated. In addition, in order to gain further insight into the interactions between thiostrepton and FOXM1, computational simulations were performed. Collectively, these data may reveal the mechanism through which thiostrepton inhibits the transactivation activity of FOXM1 and help design a novel, effective molecular inhibitor of FOXM1 in breast cancer treatment. Furthermore, a deeper understanding of the binding mechanism between thiostrepton and FOXM1 may also aid in the rational structure-based design of drug candidates.

\section{Materials and methods}

Cell culture and thiostrepton treatment. The MCF-7 breast cancer cell line was used in the present study, originating from the American Type Culture Collection and acquired through the Cancer Research UK Cell Bank. The triple-negative breast cancer MDA-MB-436 cell line was obtained from the National Nanotechnology Centre (NANOTEC) in Thailand. Both cell types were cultured in Dulbecco's modified Eagle's medium supplemented with $10 \%$ foetal bovine serum, and $100 \mathrm{U} / \mathrm{ml}$ penicillin/streptomycin (all from Gibco; Thermo Fisher Scientific, Inc.). The maintenance conditions were at $37^{\circ} \mathrm{C}$ in a humidified incubator with $5 \% \mathrm{CO}_{2}$, as previously described (4). Thiostrepton (Sigma-Aldrich; Merck KGaA) was used for cell treatment at various final concentrations, ranging between 0 and $100 \mu \mathrm{g} / \mathrm{ml}$.

MTT assay. The cell viability MTT assay was used to measure the cytotoxic effect of thiostrepton on MCF-7 or MDA-MB-436 cells after treatment for 24,48 and $72 \mathrm{~h}$. In brief, cells were seeded in a 96 -well plate at a density of 2,000 cells/well. After $24 \mathrm{~h}$, the culture medium was replaced with various concentrations of thiostrepton. At each time-point, the viability of the tested cells was determined by adding MTT (Sigma-Aldrich; Merck KGaA) and incubating at $37^{\circ} \mathrm{C}$, allowing formazan crystals to form. The crystals were dissolved in dimethyl sulfoxide (Merck KGaA) and the absorbance at $490 \mathrm{~nm}$ was measured with a microplate reader (BioTek PowerWave XS; BioTek Instruments, Inc.). Five replicate wells were used for each analysis, and three independent experiments were performed.

Reverse transcription-polymerase chain reaction (RT-PCR) analysis. MCF-7 and MDA-MB-436 cells were cultured and treated with various concentrations of thiostrepton, similar to the MTT assay. Total RNA was extracted from the cells using Ribospin $^{\mathrm{TM}}$ kit (GeneAll) and the concentration was measured using NanoDrop (DeNovix). Gene expression analyses were conducted using $2 \mathrm{X}$ HyperScript ${ }^{\mathrm{TM}}$ One-step RT-PCR Master mix (GeneAll). The forward and reverse primers (Macrogen, Inc.) were as follows $(23,24)$ : FOXM1 (all isomers a, b and c), forward 5'-CACCCCAGTGCCAACCGCTACTTG-3' and reverse 5'-AAAGAGGAGCTATCCCCTCCTCAG-3'; cyclin $\mathrm{B} 1(C C N B 1)$, a downstream target gene, forward 5'-AAG AGCTTTAAACTTTGGTCTGGG-3' and reverse 5'-CTT TGTAAGTCCTTGATTTACCATG-3'; and $\beta$-actin (ACTB), serving as a housekeeping control, forward 5'-ATCTGGCAC CACACCTTCTACAATGAGCTGCG-3' and reverse 5'-CGT 


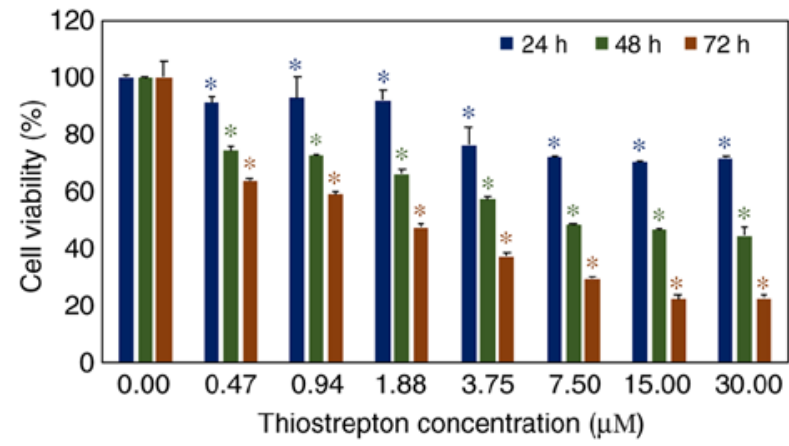

Figure 1. Thiostrepton decreases MCF-7 cell viability in dose- and timedependent manners. MCF-7 cells were treated with two-fold dilutions of thiostrepton at concentrations ranging from 0 to $30 \mu \mathrm{M}$ for 24 (blue), 48 (green) and 72 (red) h. Cell viability was measured by an MTT assay. Representative data from three independent experiments are presented. Statistical analyses were performed using one-way ANOVA with post hoc Dunnett's test and compared with the control $(0 \mu \mathrm{M})$. ${ }^{*} \mathrm{P}<0.05$ was considered as statistically significant.

CATACTCCTGCTTGCTGATCCACATCTGC-3'. The PCR conditions for all genes were 30 cycles at $96^{\circ} \mathrm{C}$ for $30 \mathrm{sec}$, annealing at $56^{\circ} \mathrm{C}$ for $30 \mathrm{sec}$ and extension at $72^{\circ} \mathrm{C}$ for $30 \mathrm{sec}$.

Senescence-associated $\beta$-galactosidase (SA- $\beta$-gal) assay. Cells were seeded in 6-well plates at a density of $10,000 \mathrm{cells} / \mathrm{well}$, then treated with thiostrepton at concentrations of $0-4 \mu \mathrm{M}$ for $24 \mathrm{~h}$ before changing to a drug-free medium, and incubated for another $24 \mathrm{~h}$. To harvest, the cells were fixed and stained using a Senescence-associated- $\beta$-galact osidase Staining Kit (cat. no. 9860, Cell Signaling Technology, Inc.), as previously described (4). The plate was incubated in a dry incubator overnight at $37^{\circ} \mathrm{C}$ without $\mathrm{CO}_{2}$. The cells that were SA- $\beta$-gal-positive were indicated by blue staining. The images were captured under a converted bright-field microscope (Nikon Eclipse TS100; Nikon Corporation) with a Nikon digital sight DS-L2 monitor (Nikon Corporation) for at least 3-5 fields. The percentages of SA- $\beta$-gal-positive cells were quantified by the amount of positive stained cells divided by the total number of cells counted.

Molecular modelling and simulations. To investigate the effects of thiostrepton on the stability of the FOXM1-DNA-binding domain, atomistic molecular dynamics (MD) simulations of the FOXM1-DNA-binding domain and thiostrepton were performed. The structures of FOXM1-DNA (25) and thiostrepton (26) were obtained from the Protein Data Bank with accession nos. 3G73 (https://www.rcsb.org/structure/3G73) and $2 \mathrm{~L} 2 \mathrm{~W}$ (https://www.rcsb.org/structure/2L2W), respectively. The isolated FOXM1-DNA-binding domain was expressed in E. coli and the 3D structure was determined by X-ray diffraction with a resolution of 2.21 Ångström (25). The DNA double-strand target consisted of a DNA-A and a DNA-B chain, with a base-paired DNA sequence of AAATTG TTTATAAACAGCCCG and TTCGGGCTGTTTATAAAC AAT for DNA-A and DNA-B, respectively. For thiostrepton, the molecular structure was determined by nuclear magnetic resonance (26). The topology of thiostrepton was created by a web-accessible Automated force field Topology Builder (ATB; http://compbio.biosci.uq.edu.au/atb/) (27-29). Initially, the protein structures of FOXM1-DNA and thiostrepton were submitted to the AutoDockTools-1.5.6 (Autodock-4.2) (30) to search the binding region. To identify the thiostrepton-binding complex structure, three different models were performed, including i) a FOXM1 monomer, ii) a FOXM1 dimer and iii) a FOXM1 dimer complex with DNA. To study the structure stability of the binding complex, the structure with thiostrepton in the binding domain region of the FOXM1 dimer model was selected for further MD simulations. The details of the selected structures are discussed in Results and Discussion.

Two MD simulations of FOXM1-DNA with and without thiostrepton were considered in this study and carried out using GROMACS version 5.1.3 (31). The structure of thiostrepton at the lowest binding energy in the FOXM1 dimer model was used as the initial structure. The parameters of all molecules were derived from GROMOS53a6 force field (32) and the simple point-charge (SPC) model (33) was used for water. Each system was solvated by 37,337 water molecules, and $17 \mathrm{Mg}^{2+}$ ions were added for neutralizing the system charges. The system energy was minimized by the steepest descent algorithm to remove steric conflicts between atoms. In order to avoid rearrangement of the FOXM1 protein structures, position restraints were applied to the FOXM1 backbone with a force constant of $1,000 \mathrm{~kJ}(\mathrm{~mol} \mathrm{~nm})^{-1}$ in all directions. Subsequently, MD simulations were performed under NPT (constant particle number, pressure and temperature) ensemble for $200 \mathrm{nsec}$ with an integration time step of $1 \mathrm{fsec}$. The last $50 \mathrm{nsec}$ of the trajectories were used to determine the binding interactions among FOXM1, DNA and thiostrepton. The temperature was maintained constant using the velocity-rescale (V-rescale) algorithm $(34,35)$ at $298 \mathrm{~K}$ with a time constant of $0.1 \mathrm{psec}$. Semi-isotropic pressure was applied under a constant pressure of 1 bar with a time constant of $3.0 \mathrm{psec}$ and a compressibility of $4.5 \times 10^{-5}$ bar $^{-1}$ using the Parrinello-Rahman algorithm (36). Periodic boundary conditions in all directions were used. A cut-off with a distance of $1.0 \mathrm{~nm}$ was applied for the real-space part of the electrostatic and Lennard-Jones interaction. In the reciprocal-space interactions of long-range electrostatics, the Particle-mesh Ewald $(37,38)$ method was employed with a $0.12-\mathrm{nm}$ grid and the cubic interpolation of order four. The simulation protocol had been tested to avoid physical artefacts (39). All bond lengths were constrained using the P-LINCS algorithm (40). The Visual Molecular Dynamics (VMD) software was used for system visualization (41).

Statistical analysis. All statistical analyses were performed using SPSS 16.0 (Imperial College London) and Microsoft Excel 2013 (Kasetsart University). Values are presented as the mean \pm standard deviation (SD). One-way ANOVA was performed followed by post hoc analysis with Dunett's test. P-values $<0.05$ were considered to indicate statistically significant differences.

\section{Results and Discussion}

In vitro investigation of thiostrepton suppresses breast cancer cell growth. The effect of thiostrepton on the suppression of MCF-7 breast cancer cell growth was studied using an MTT assay. The results revealed that thiostrepton significantly restricted cancer cell proliferation in a dose-dependent manner. 

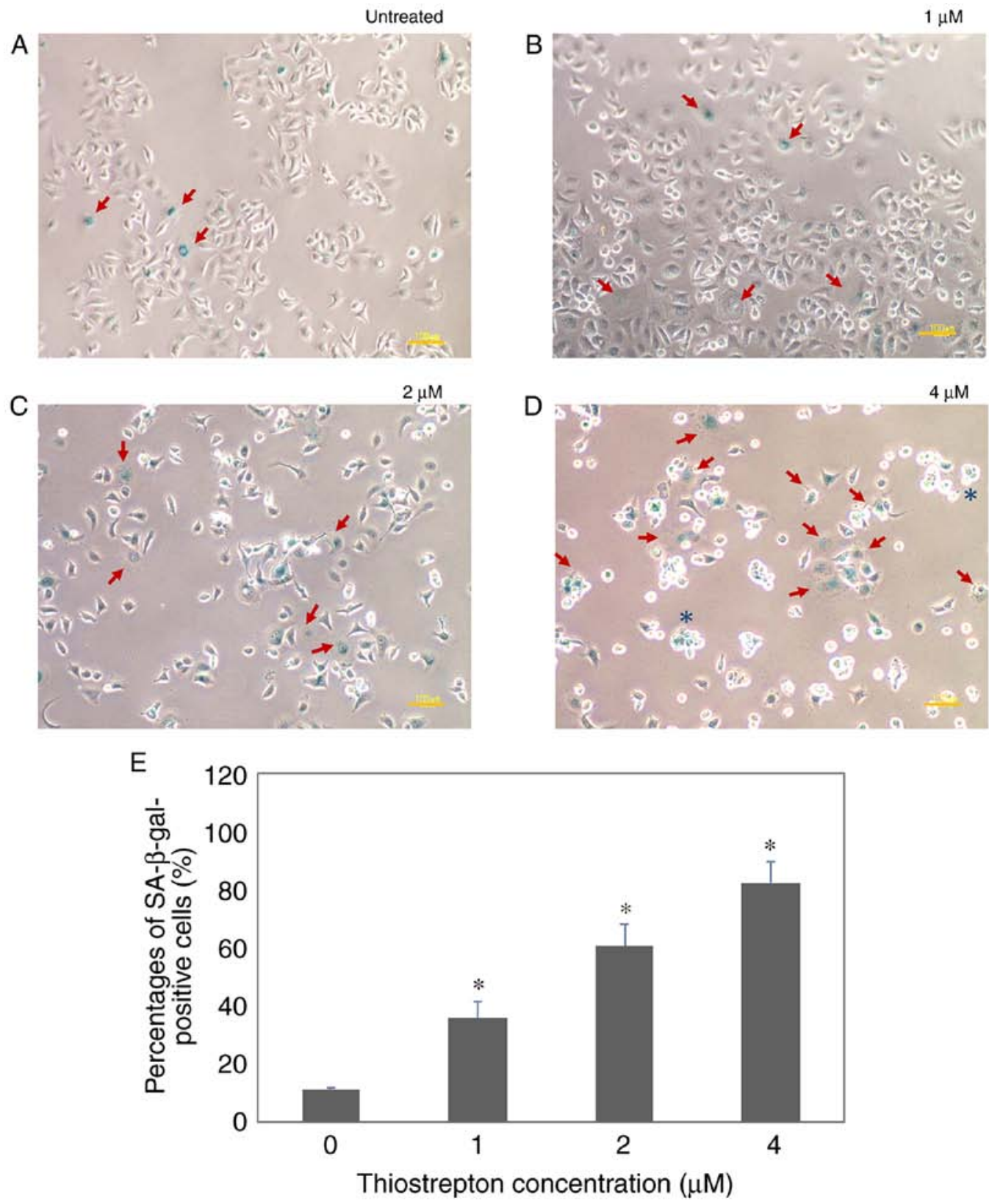

Figure 2. Thiostrepton treatment at low concentrations induces cellular senescence in MCF-7 cells. SA- $\beta$-gal staining of (A) untreated cells, or cells treated with thiostrepton at concentrations of (B) $1 \mu \mathrm{M}$, (C) $2 \mu \mathrm{M}$ and (D) $4 \mu \mathrm{M}$ for $24 \mathrm{~h}$. The treated cells were stained for SA- $\beta$-gal activity (green, SA- $\beta$-gal-positive). Red arrows indicate morphological changes in senescent cells. Blue asterisks, morphological changes in dying cells. (E) The percentages of SA- $\beta$-gal-positive cells are presented as the mean \pm standard deviation. Statistical analyses were performed using one-way ANOVA with post hoc Dunnett's test and compared with the control $(0 \mu \mathrm{M}) .{ }^{*} \mathrm{P}<0.05$ was considered as statistically significant. SA- $\beta$-gal senescence-associated $\beta$-galactosidase.

Following thiostrepton exposure for $24 \mathrm{~h}$, cell viability was significantly decreased $(\mathrm{P}<0.05)$ after being treated with a dose of $0.47 \mu \mathrm{M}$ (Fig. 1). After 48 and $72 \mathrm{~h}$ of treatment, the viability of MCF-7 cells was significantly reduced by lower doses of thiostrepton $(0.47 \mu \mathrm{M})$. Additionally, the effect of thiostrepton on the triple-negative breast cancer cell line MDA-MB-436 was similar to that on MCF-7 cells (Fig. S1). Both breast cancer cell types were affected in a dose-dependent manner. These results confirmed that thiostrepton is an effective candidate compound for developing a novel anticancer treatment (11).

Thiostrepton potently induces senescence in MCF-7 breast cancer cells. Inhibition of FOXM1 has been shown to induce cell cycle arrest due to its crucial role in G1 and G2/M cell cycle phase progression. Depletion of FOXM1 results in DNA damage and induces cellular senescence (4). Therefore, we investigated whether the effects of FOXM1 inhibition by thiostrepton treatment are mediated by causing irreversible cell cycle arrest (also referred to as cellular senescence). MCF-7 cells were assayed for SA- $\beta$-gal positivity after the cells were subjected to a sequence of thiostrepton concentrations $(0,1$, 2 and $4 \mu \mathrm{M}$ ), as revealed in Fig. 2. Senescent cells displayed SA- $\beta$-gal activity and were stained green in the presence of chromogenic substrate 5-bromo-4-chloro-3-indolyl- $\beta$-d-galac topyranoside (X-gal). Consistently, MCF-7 cells treated with thiostrepton exhibited SA- $\beta$-gal activity and their morphology changed (Fig. 2B-D, red arrows), with the cells becoming enlarged and flattened. This was consistent with our previous 


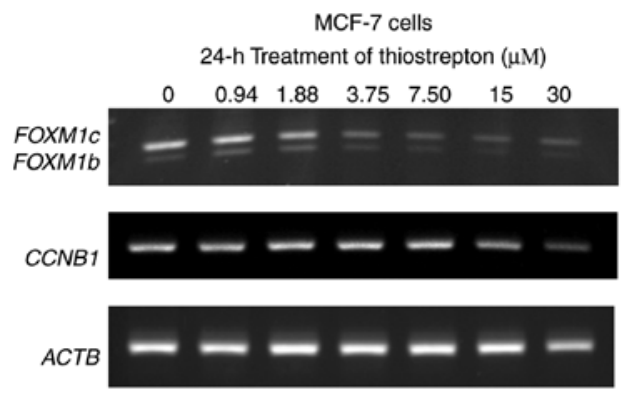

Figure 3. Thiostrepton treatment for $24 \mathrm{~h}$ downregulates the expression of FOXM1 and its downstream target in MCF-7 cells. The RT-PCR analysis of mRNA expression using the primers detected the isoforms FOXM1c (1,039 bp) and FOXM1b (996 bp). Cyclin B1 and FOXM1 (auto-regulated) were the main downstream targets of the FOXM1 protein, and $A C T B$ served as the internal control. $C C N B 1$, cyclin B1; ACTB, $\beta$-actin; FOXM1, Forkhead box M1.

findings on cell cycle analysis, demonstrating that thiostrepton can cause cell cycle arrest at the G1 phase in MCF-7 cells, but not in untransformed breast epithelial MCF-10 cells (11). The SA- $\beta$-gal activity was determined to be increased in a dose-dependent manner, with the highest activity observed at $4 \mu \mathrm{M}$. At this concentration, some cells also became rounded, which is consistent with the morphology of dying cells (Fig. 2D, blue asterisk). In addition, MDA-MB-436 cells also exhibited senescence morphology, with positive SA- $\beta$-gal activity, after being exposed to thiostrepton (Fig. S2). These thiostrepton-treated cells displayed typical senescence characteristics, such as enlargement, flattening and reduction in numbers $(13,14)$. Under the bright-field microscope, additional cell death morphological characteristics, such as vacuolization, were also observed. Moreover, the cells started to detach from the culture plate and assume a rounder shape. Of note, the cells incubated with 2 and $4 \mu \mathrm{M}$ of thiostrepton exhibited lower viability, as some of the cells died after treatment (Fig. 2). These findings highlight the ability of thiostrepton to induce senescence in breast cancer cells. The results strongly indicated that thiostrepton can inhibit cancer progression by inducing both cell death and senescence via a FOXM1-dependent pathway.

Thiostrepton reduces FOXM1 and CCNB1 expression at the transcriptional level. Next, the effect of thiostrepton on mRNA expression of FOXM1 (auto-regulation) (42) and the well-known FOXM1 downstream target $C C N B 1$ (43) were investigated. After $24 \mathrm{~h}$ of thiostrepton exposure, MCF-7 cells exhibited downregulation of FOXM1 (Fig. 3). The decrease in FOXM1 levels was evident at a thiostrepton concentration of $3.75 \mu \mathrm{M}$. This downregulation of FOXM1 mRNA may be attributed to the auto-regulation ability of FOXM1, as it can bind to its own promoter and regulate its transcription $(18,42)$. For $C C N B 1$, the mRNA expression was decreased after thiostrepton treatment at $15 \mu \mathrm{M}$. Of note, the FOXM1 and CCNBI mRNA levels decreased with increasing drug concentrations (Fig. 3) and time of exposure (Fig. S3), which were also correlated with significant decreases in the viability of MCF-7 cells (Fig. 3). Notably, the great changes on the CCNBI mRNA level at $30 \mu \mathrm{M}$ indicated the effect of transactivation activity of thiostrepton on FOXM1. A similar phenomenon was also observed in MDA-MB-436 cells. The mRNA expression of FOXM1 and $C C N B 1$ was also decreased after thiostrepton exposure
A

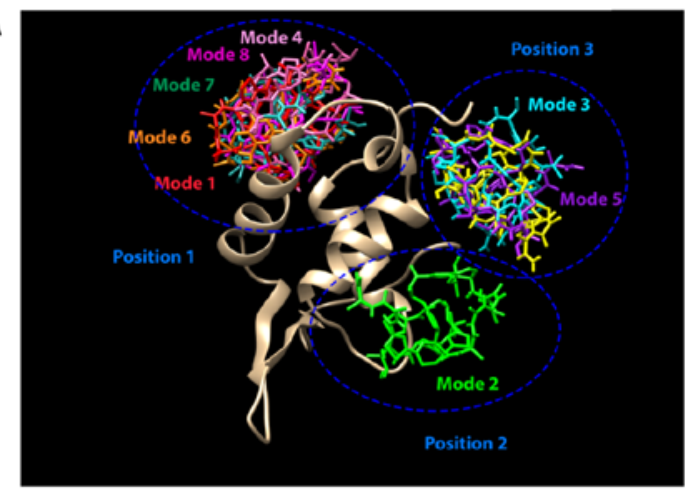

B

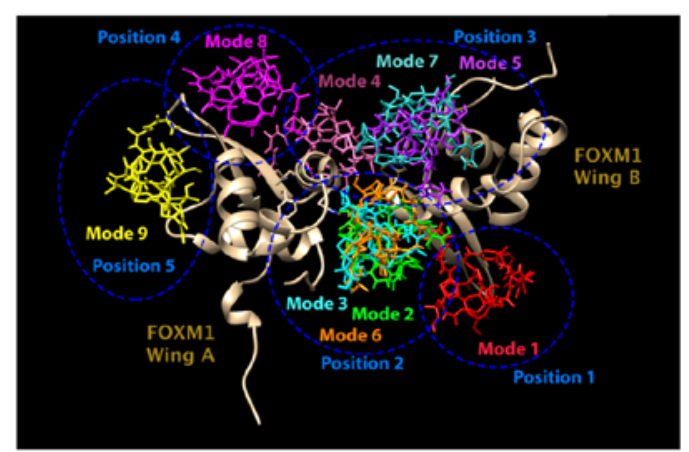

C

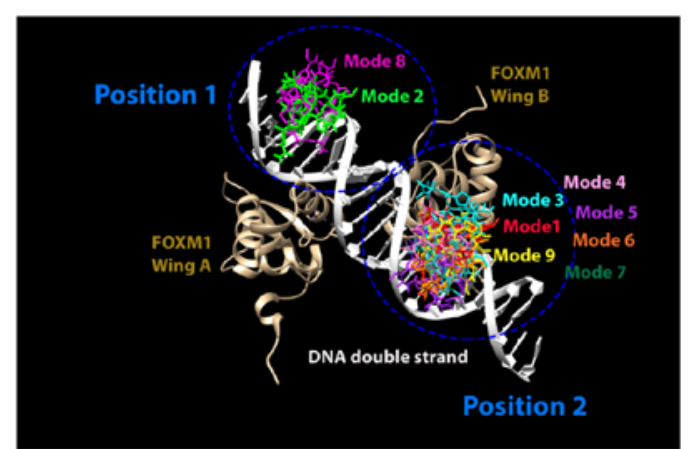

Figure 4. Different modes of thiostrepton binding to FOXM1 (golden bronze) with different models: (A) Monomer FOXM1, (B) dimer FOXM1 and (C) FOXM1-DNA complex. The lowest binding energy of thiostrepton was found in mode 1 (red), followed by mode 2 (green), 3 (cyan), 4 (pink), 5 (purple), 6 (orange), 7 (sea blue-green), 8 (magenta) and 9 (yellow). Blue circles, different possible positions of binding area. FOXM1, Forkhead box M1.

in dose- and time-dependent manners (Figs. S3 and S4). Collectively, these data indicated that thiostrepton inhibited FOXM1 mRNA expression for both isoforms $\mathrm{b}$ and $\mathrm{c}$. These two isoforms of FOXM1 play a crucial role in carcinogenesis, cancer progression and metastasis $(44,45)$, and the downregulation of FOXM1 mRNA expression appears to be an interesting target for anticancer therapy $(18,46)$. Therefore, our results suggest that thiostrepton may be a good candidate for inhibition of cancer cell growth and metastasis in patients with FOXM1 overexpression $(12,18,46)$.

Interactions of thiostrepton and binding domain of FOXM1 forkhead/winged-helix domain (FKH) with targeted DNA promoter motif. In a previous study (25), the FOXM1 forkhead/winged helix domain (FKH) sequence at residues 222-360 was identified as the DBD with a tandem recogni- 


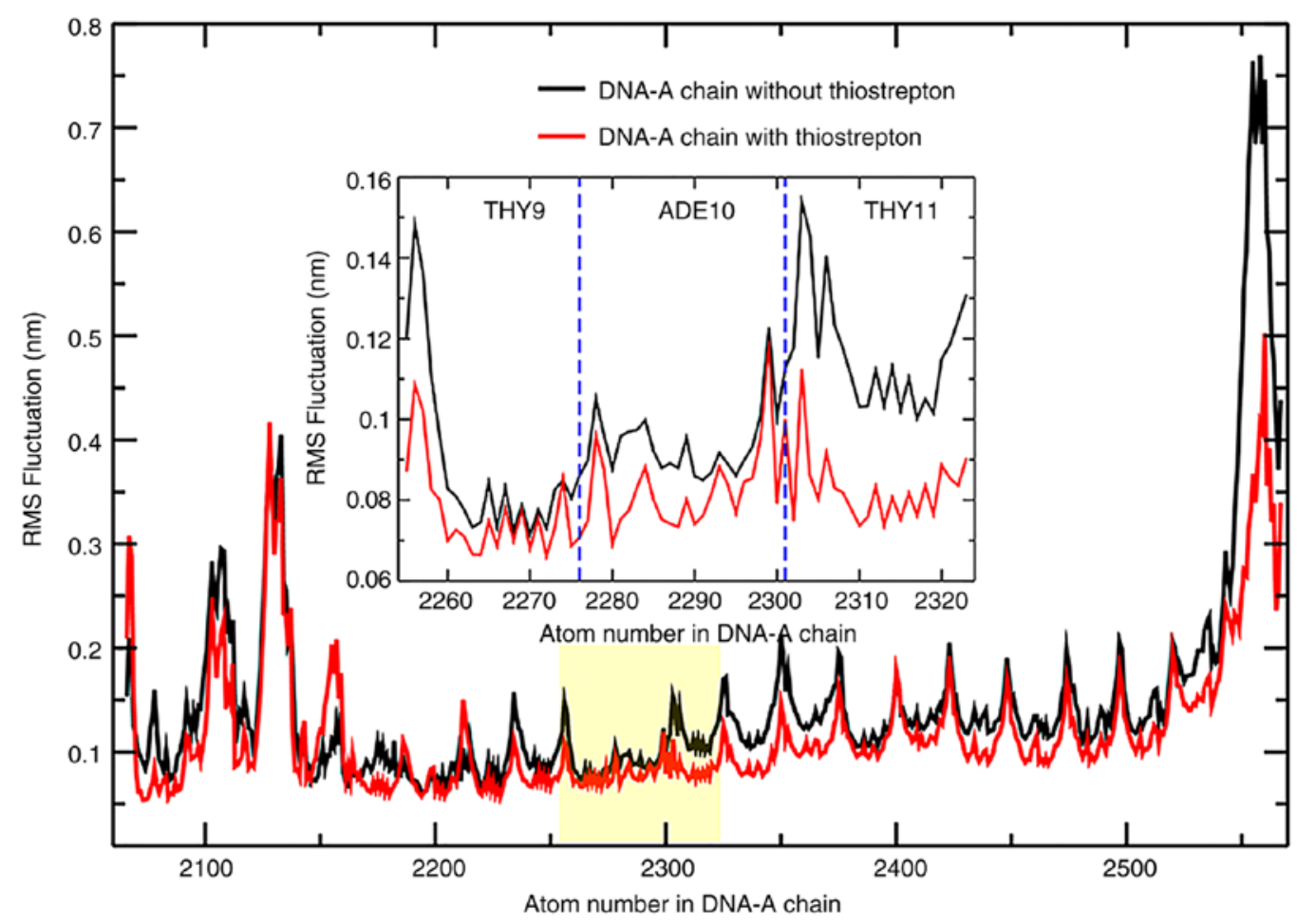

Figure 5. The RMSF of the DNA-A chain forms a complex with the FOXM1 dimer. The systems with and without thiostrepton in the DNA-A-binding domain are represented in red and black lines, respectively. Inset shows the RMSF in specific binding residues of Thy9, Ade10 and Thy11 of the DNA-A chain. The numbers 9, 10 and 11 refer to the order of base-paired sequence in the DNA-A chain. RMSF, root-mean-square fluctuation; FOXM1, Forkhead box M1.

tion sequence of the downstream target (TAAACA) (47). Specific mutations in the DBD have been revealed to lead to the decrease of FOXM1 transcriptional activity (48). The binding energy (affinity) between FOXM1 and DNA promoter motif were calculated by the molecular docking technique (30). FOXM1 was selected as the protein macromolecule and the DNA promoter motif served as the ligand. The nine complex structures with different binding modes were used to calculate the binding energy $(\Delta \mathrm{G})$, with mode 1 having the lowest binding energy. The $\Delta \mathrm{G}$ between FOXM1 and promoter DNA motif was determined to be between - 6.0 and $-5.3 \mathrm{kcal} / \mathrm{mol}$, in agreement with the preferential binding of the FOXM1 to the targeted promoter motif DNA sequence. Next, the thiostrepton binding affinity to FOXM1 was determined by performing three different models, as revealed in Fig. 4: i) FOXM1 monomer model, ii) FOXM1 dimer model and iii) FOXM1 dimer complex with DNA. The first model (FOXM1 monomer) revealed that thiostrepton could bind to the DBD at three possible positions and the binding energy of this model was in the range of -6.4 to $-5.5 \mathrm{kcal} / \mathrm{mol}$ (Fig. 4A). For the second model (FOXM1 dimer; wing A and wing B), five possible positions between thiostrepton and the FOXM1 DBD proteins were found, with the binding energy ranging from -7.8 to $-6.9 \mathrm{kcal} / \mathrm{mol}$ (Fig. 4B). The last model (FOXM1 dimer complex with DNA binding motif) revealed that the binding affinity was decreased $(-8.0$ to $-7.6 \mathrm{kcal} / \mathrm{mol})$, with two possible positions (Fig. 4C); however, neither position was suitable to accommodate thiostrepton inside the binding domain of FOXM1. Conversely, the first and second models enabled thiostrepton to prevent the binding of FOXM1 to the DNA motif. Chen et al (21) previously investigated the notable behavior of thiostrepton in contact with the FOXM1 dimer and partial binding to the DNA motif. Therefore, the thiostrepton structure in the FOXM1 dimer from the second model (Mode 1 in Fig. 4B) in a complex with DNA binding motif was selected to perform molecular dynamic (MD) simulations in order to compare to the structure without thiostrepton. The root-mean-square fluctuation (RMSF) analysis indicated a stabilized structure of DNA, particularly at residues in the binding domain regions of FOXM1 (Fig. 5). This result demonstrated that thiostrepton can increase the stability of the binding between DNA and FOXM1 inside the DBD.

The mean number and lifetime of hydrogen bonds among FOXM1, DNA and thiostrepton were analyzed, as it was previously suggested that FOXM1-DNA complex interaction involves van der Waals forces and hydrogen bonding (49). A hydrogen bond was formed when the distance between donor and acceptor $\left(\mathrm{r}_{\mathrm{HB}}\right)$ was $<0.35 \mathrm{~nm}$ and the hydrogen-donor-acceptor angle $\left(\alpha_{\mathrm{HB}}\right)$ was $<30^{\circ}$. The value of $0.35 \mathrm{~nm}$ corresponds to the first minimum of the radial distribution function of water. The overall hydrogen bond lifetime $\left(\tau_{\mathrm{HB}}\right)$ is calculated from the mean over all autocorrelation functions $C(\tau)$ of the lifetime distribution $P(\tau)$ of all hydrogen bonds from time 0 to $t$ : (50):

$$
\begin{aligned}
& C(\tau)=1-\int_{0}^{t} P(\tau) d \tau \\
& \tau_{H B}=\int_{0}^{\infty} C(\tau) d \tau
\end{aligned}
$$




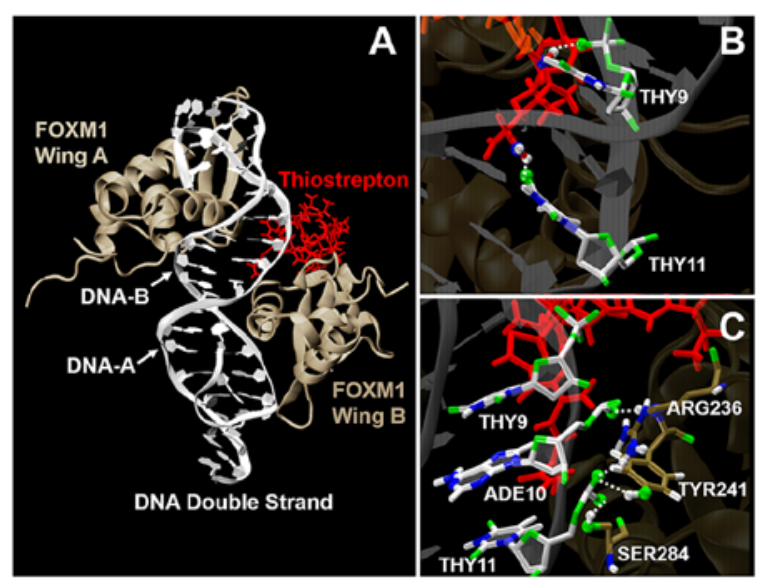

Figure 6. (A) The final structure at $200 \mathrm{nsec}$ of the system containing FOXM1-DNA with thiostrepton (red). The FOXM1 dimers are represented in golden bronze. DNA double-strand target consists of the DNA-A chain [AAATTGTTTATAAACAGCCCG] and the DNA-B chain [TTCGGGCTGTTTATAAACAAT], presented in white. (B) Thy 9 and Thy11 in DNA-A formed hydrogen bonds with thiostrepton. (C) Four hydrogen bonds were found between the residues of FOXM1 and DNA-A inside the DNA-binding domain. FOXM1, Forkhead box M1.

The number of hydrogen bonds in the binding promoter region among FOXM1 wing A, DNA-A and thiostrepton were analyzed and are presented in Table I and Fig. 6. The hydrogen bond binding domains were observed in residues Tyr241(tyrosine), Ser284(serine) and Arg236 (arginine) of the FOXM molecule, and residues Ade10 (adenine) and Thy11 (thymine) of the DNA-A chain. These binding residues were consistent with those reported previously (25). The number of hydrogen bonds between FOXM1 wing A and DNA-A in the system was $4.34 \pm 0.24$ and decreased slightly to $4.17 \pm 0.05$ when the thiostrepton molecule formed a complex with the FOXM1-DNA binding domain. Moreover, the increase in binding stability may be explained by the presence of $0.95 \pm 0.66$ hydrogen bonds between thiostrepton and DNA-A in the promoter binding region. In addition, thiostrepton forming a complex with FOXM1-DNA was associated with an increase in hydrogen bond lifetime in the binding domain. The mean number of hydrogen bonds and their lifetimes are presented in Table I. Notably, our results indicated that the increase in the stability of hydrogen bonds in the promoter binding region may result in transcription inhibition. Moreover, these results also indicated a mechanism through which thiostrepton inhibits the transcriptional activity of a transcription factor. However, protein-DNA interaction experiments such as chromatin immunoprecipitation sequencing (ChIP) assay, electrophretic mobility shift assay, and reporter assay may be performed in a future study to confirm the experimentally assessable potential of FOXM1-DNA interaction in vitro.

Clinically, FOXM1 expression has been confirmed to be significantly associated with resistance to chemotherapy and suggested to be a reliable biomarker for adverse prognosis in cancer patients (51). Conversely, depletion of FOXM1 was revealed to lead to the decrease of homologous recombination repair in breast cancer cells and the increase in sensitivity to genotoxic chemotherapy drugs (5). Three isoforms of the FOXM1 protein exist, and they are the result of alternative

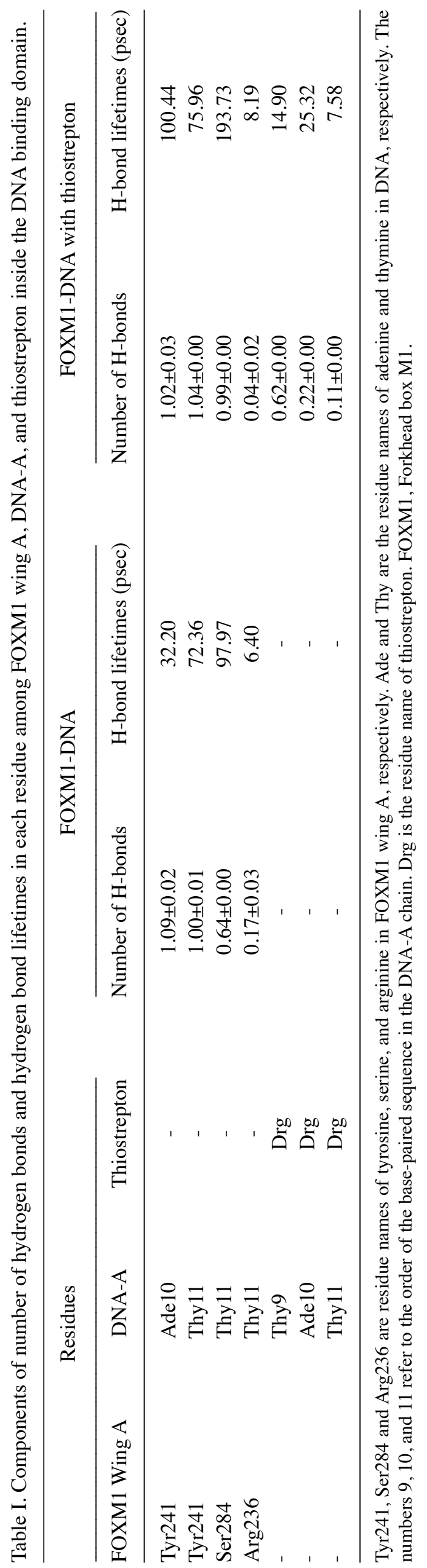


splicing (6). FOXM1a is entirely inactive, as both alternative exons insert in the $\mathrm{C}$-terminal transactivation domain $(6,9,52)$. By contrast, the other two isoforms, FOXM1b and FOXM1c, are transcriptionally active and responsible for cancer cell growth and resistance to chemotherapy by promoting the expression of genes involved in cell proliferation, DNA repair and other characteristics important for cancer development and progression (6).

In the present study, the effect of thiostrepton on cell viability was examined by using an MTT cell viability assay and RT-PCR. The results confirmed that thiostrepton alone was able to reduce the percentage of viable breast cancer cells by suppressing the ability of FOXM1 to regulate the expression of target genes, including FOXM1 and CCNB1, at the transcriptional level. Moreover, the present study also demonstrated that thiostrepton can inhibit the transcriptional activity of both FOXM1b and FOXM1c, the dominant isoforms in various types of cancer $(6,53)$. These results indicated that thiostrepton has the potential to be used in both FOXM1b- and FOXM1c-overexpressing cancer patients. In addition, thiostrepton has been reported to promote cancer cell death by apoptosis and by inducing cell cycle arrest at the G1/S phase (11). The status of the cell cycle was further investigated and it was revealed that the cycle arrest of breast cancer cells induced by thiostrepton is an irreversible event via cellular senescence $(4,13,14)$. The present results demonstrated that senescent cancer cells characteristically change their shape and size $(13,14)$. Although thiostrepton was clearly revealed to decrease cell viability and induce cellular senescence, the molecular mechanism underlying inhibition of FOXM1 expression remains controversial $(15,16)$ Previous studies demonstrated that a small-molecule inhibitor, such as FDI-6, may interfere with the binding of DBD and the targeted DNA, resulting in the inhibition of FOXM1 function and transcriptional programing (49,54-56) Notably, our molecular docking results also demonstrated the ability of thiostrepton to bind to the FOXM1-DNA complex, thereby interfering with the transcription of the downstream targets. This evidence further confirms that thiostrepton is a suitable compound for developing FOXM1-targeted therapy, as it can directly and specifically bind to FOXM1 $(11,16,21)$ The MD simulation demonstrated that the lifetime of hydrogen bonds between wing-helix DBD FOXM1 and the targeted DNA motif can be increased by forming complexes with thiostrepton. Changes in interaction between protein and DNA, such as a tight binding, will cause unfavorable conditions for binding and sliding between protein and DNA (57). Since FOXM1 is an autoregulated protein, the increasing stability of the hydrogen bonds may play a key role in suppressing the activity of FOXM1 and leading to downregulation of FOXM1.

Collectively, our findings may enable a better understanding of the DNA-FOXM1 binding with thiostrepton and may aid in developing future anticancer strategies. In silico and in vitro studies help to verify the efficiency of the anticancer effects of thiostrepton at an atomic level (25). In agreement with previous studies $(11,17,22)$, we herein demonstrated that thiostrepton may be an optimal compound for overcoming cancer resistance to chemotherapy (particularly to cisplatin, carboplatin, epirubicin and doxorubicin) via targeting FOXM1 $(11,58)$.

\section{Acknowledgements}

The authors would like to thank Associate Professor Kiattawee Choowongkomon, Department of Biochemistry, Faculty of Science, Kasetsart University, for the valuable advice and ideas during the research process. Computing facilities were provided by the Department of Physics, Faculty of Science, Kasetsart University.

\section{Funding}

The present was supported by a grant from Kasetsart University Research and Development Institute (KURDI), Kasetsart University, Bangkok, Thailand. SW received a grant by the National Research Council of Thailand (NRCT: Graduate Scholarship, 2019) and the Thailand Graduate Institute of Science and Technology (TGIST: grant no. SCA-CO-2561-6950-TH) affiliated to the National Science and Technology Development Agency (NSTDA). The support of the Thailand Research Fund through the Royal Golden Jubilee Ph.D. Program (grant no. PHD/0204/2559) and the TRF Research Scholar (grant no. RSA6180021) to PB and JW is acknowledged. The research of EW-FL was supported by the Medical Research Council (MRC) (MR/N012097/1), Cancer Research UK (CRUK) (C37/A12011;C37/A18784), Breast Cancer Now (2012MayPR070; 2012NovPhD016), the Cancer Research UK Imperial Centre, the Imperial Experimental Cancer Medicine Centre (ECMC) and the National Institute for Health Research (NIHR) Imperial Biomedical Research Centre (BRC).

\section{Availability of data and materials}

The datasets used during the present study are available from the corresponding author upon reasonable request.

\section{Authors' contributions}

MKo designed and performed the experiments, analysed and the interpreted data. SW performed the in vivo experiments and the statistical analysed the data. MKh designed and interpreted the data. EWFL designed the experiments, analysed and provided some critical idea during analyses. WV analysed and interpreted the data. PB and JWE designed, performed the MD simulations and analysed the data. All authors read and approved the final manuscript and agree to be accountable for all aspects of the research in ensuring that the accuracy or integrity of any part of the work are appropriately investigated and resolved.

\section{Ethics approval and consent to participate}

Not applicable.

\section{Patient consent for publication}

Not applicable.

\section{Competing interests}

The authors declare that they have no competing interests. 


\section{References}

1. Krebs MG, Hou JM, Ward TH, Blackhall FH and Dive C: Circulating tumour cells: Their utility in cancer management and predicting outcomes. Ther Adv Med Oncol 2: 351-365, 2010

2. Thorn CF, Oshiro C, Marsh S, Hernandez-Boussard T, McLeod H, Klein TE and Altman RB: Doxorubicin pathways: Pharmacodynamics and adverse effects. Pharmacogenet Genomics 21: 440-446, 2011

3. Myatt SS, Kongsema M, Man CW, Kelly DJ, Gomes AR, Khongkow P, Karunarathna U, Zona S, Langer JK, Dunsby CW, et al: SUMOylation inhibits FOXM1 activity and delays mitotic transition. Oncogene 33: 4316-4329, 2014.

4. Khongkow P, Karunarathna U, Khongkow M, Gong C, Gomes AR, Yagüe E, Monteiro LJ, Kongsema M, Zona S, Man EP, Tsang JW, et al: FOXM1 targets NBS1 to regulate DNA damage-induced senescence and epirubicin resistance. Oncogene 33: 4144-4155, 2014.

5. Monteiro LJ, Khongkow P, Kongsema M, Morris JR, Man C, Weekes D, Koo CY, Gomes AR, Pinto PH, Varghese V, et al: The Forkhead Box M1 protein regulates BRIP1 expression and DNA damage repair in epirubicin treatment. Oncogene 32: 4634-4645, 2013.

6. Koo CY, Muir KW and Lam EW: FOXM1: From cancer initiation to progression and treatment. Biochim Biophys Acta 1819: 28-37, 2012.

7. Millour J, de Olano N, Horimoto Y, Monteiro LJ, Langer JK, Aligue R, Hajji N and Lam EW: ATM and p53 regulate FOXM1 expression via $\mathrm{E} 2 \mathrm{~F}$ in breast cancer epirubicin treatment and resistance. Mol Cancer Ther 10: 1046-1058, 2011.

8. Karunarathna U, Kongsema M, Zona S, Gong C, Cabrera E, Gomes AR, Man EP, Khongkow P, Tsang JW, Khoo US, et al: OTUB1 inhibits the ubiquitination and degradation of FOXM1 in breast cancer and epirubicin resistance. Oncogene 35: 1433-1444, 2016.

9. Laoukili J, Stahl M and Medema RH: FoxM1: At the crossroads of ageing and cancer. Biochim Biophys Acta 1775: 92-102, 2007.

10. Nicolaou KC, Zak M, Rahimipour S, Estrada AA, Lee SH, O'Brate A, Giannakakou P and Ghadiri MR: Discovery of a biologically active thiostrepton fragment. J Am Chem Soc 127: 15042-15044, 2005

11. Kwok JM, Myatt SS, Marson CM, Coombes RC, Constantinidou D and Lam EW: Thiostrepton selectively targets breast cancer cells through inhibition of forkhead box M1 expression. Mol Cancer Ther 7: 2022-2032, 2008

12. Ahmed M, Hussain A, Begum R, Thangavel S, Ajarim DS, Beg S, Uddin S and Al-Kuraya KS: Abstract 55: Over-expression of FoxM1 in breast cancer can be therapeutically targeted using thiostrepton. Cancer Res 75: 55-55, 2015.

13. Collado M and Serrano M: Senescence in tumours: Evidence from mice and humans. Nat Rev Cancer 10: 51-57, 2010.

14. Rodier F and Campisi J: Four faces of cellular senescence. J Cell Biol 192: 547-556, 2011.

15. Gartel AL: Thiostrepton, proteasome inhibitors and FOXM1. Cell Cycle 10: 4341-4342, 2011.

16. Hegde NS, Sanders DA, Rodriguez R and Balasubramanian S: The transcription factor FOXM1 is a cellular target of the natural product thiostrepton. Nat Chem 3: 725-731, 2011.

17. Gartel AL: Suppression of the oncogenic transcription factor FOXM1 by proteasome inhibitors. Scientifica (Cairo) 2014: 596528, 2014.

18. Gartel AL: Targeting FOXM1 auto-regulation in cancer. Cancer Biol Ther 16: 185-186, 2015.

19. Bhat UG, Halasi M and Gartel AL: Thiazole antibiotics target FoxM1 and induce apoptosis in human cancer cells. PLoS One 4: e5592, 2009.

20. Gartel AL: A new target for proteasome inhibitors: FoxM1. Expert Opin Investig Drugs 19: 235-242, 2010.

21. Chen Y, Ruben EA, Rajadas J and Teng NN: In silico investigation of FOXM1 binding and novel inhibitors in epithelial ovarian cancer. Bioorg Med Chem 23: 4576-4582, 2015.

22. Gartel AL: Thiazole antibiotics siomycin a and thiostrepton inhibit the transcriptional activity of FOXM1. Front Oncol 3 : $150,2013$.

23. Nakamura S, Hirano I, Okinaka K, Takemura T, Yokota D, Ono T, Shigeno K, Shibata K, Fujisawa S and Ohnishi K: The FOXM1 transcriptional factor promotes the proliferation of leukemia cells through modulation of cell cycle progression in acute myeloid leukemia. Carcinogenesis 31: 2012-2021, 2010.
24. Villa-Diaz LG, Garcia-Perez JL and Krebsbach PH: Enhanced transfection efficiency of human embryonic stem cells by the incorporation of DNA liposomes in extracellular matrix. Stem Cells Dev 19: 1949-1957, 2010.

25. Littler DR, Alvarez-Fernández M, Stein A, Hibbert RG, Heidebrecht T, Aloy P, Medema RH and Perrakis A: Structure of the FoxM1 DNA-recognition domain bound to a promoter sequence. Nucleic Acids Res 38: 4527-4538, 2010.

26. Jonker HR, Baumann S, Wolf A, Schoof S, Hiller F, Schulte KW, Kirschner KN, Schwalbe H and Arndt HD: NMR structures of thiostrepton derivatives for characterization of the ribosomal binding site. Angew Chem Int Ed Engl 50: 3308-3312, 2011.

27. Malde AK, Zuo L, Breeze M, Stroet M, Poger D, Nair PC, Oostenbrink C and Mark AE: An automated force field topology builder (ATB) and repository: Version 1.0. J Chem Theory Comput 7: 4026-4037, 2011.

28. Canzar S, El-Kebir M, Pool R, Elbassioni K, Mark AE, Geerke DP, Stougie L and Klau GW: Charge Group Partitioning in Biomolecular Simulation. J Comput Biol 20: 188-198, 2013.

29. Koziara KB, Stroet M, Malde AK and Mark AE: Testing and validation of the Automated Topology Builder (ATB) version 2.0: Prediction of hydration free enthalpies. J Comput Aid Mol Des 28: 221-233, 2014

30. Trott $\mathrm{O}$ and Olson AJ: Software news and update AutoDock Vina: Improving the speed and accuracy of docking with a new scoring function, efficient optimization, and multithreading. J Comput Chem 31: 455-461, 2010

31. Abraham MJ, Murtola T, Schulz R, Páll S, Smith JC, Hess B and Lindahl E: GROMACS: High performance molecular simulations through multi-level parallelism from laptops to supercomputers. SoftwareX 1-2: 19-25, 2015.

32. Oostenbrink C, Villa A, Mark AE and Van Gunsteren WF: A biomolecular force field based on the free enthalpy of hydration and solvation: The GROMOS force-field parameter sets 53A5 and 53A6. J Comput Chem 25: 1656-1676, 2004.

33. Berendsen HJC, Postma JPM, Gunsteren WF and Hermans J: Interaction models for water in relation to protein hydration. In: Intermolecular forces. Pullman B (ed). Vol 14. Springer, Berlin, pp331-334, 1981

34. Bussi G, Donadio D and Parrinello M: Canonical sampling through velocity rescaling. J Chem Phys 126: 2007.

35. Bussi G, Zykova-Timan T and Parrinello M: Isothermal-isobaric molecular dynamics using stochastic velocity rescaling. J Chem Phys 130: 074101, 2009.

36. Parrinello $\mathrm{M}$ and Rahman A: Polymorphic transitions in single crystals: A new molecular dynamics method. J Appl Phys 52, 1981.

37. Darden T, York D and Pedersen L: Particle mesh Ewald: An N I $\log (\mathrm{N})$ method for Ewald sums in large systems. J Chem Phys 98: 10089, 1993.

38. Essmann U, Perera L and Berkowitz ML: A smooth particle mesh Ewald method. J Chem Phys 103: 8577, 1995.

39. Wong-Ekkabut $\mathbf{J}$ and Karttunen M: Assessment of common simulation protocols for simulations of nanopores, membrane proteins, and channels. J Chem Theory Comput 8: 2905-2911, 2012.

40. Hess B: P-LINCS: A parallel linear constraint solver for molecular simulation. J Chem Theory Comput 4: 116-122, 2008.

41. Humphrey W, Dalke A and Schulten K: VMD: Visual molecular dynamics. J Mol Graph 14: 33-38, 27-38, 1996.

42. Halasi M and Gartel AL: A novel mode of FoxM1 regulation: Positive auto-regulatory loop. Cell Cycle 8: 1966-1967, 2009.

43. Leung TW, Lin SS, Tsang AC, Tong CS, Ching JC, Leung WY, Gimlich R, Wong GG and Yao KM: Over-expression of FoxM1 stimulates cyclin B1 expression. FEBS Lett 507: 59-66, 2001.

44. Liao GB, Li XZ, Zeng S, Liu C, Yang SM, Yang L, Hu CJ and Bai JY: Regulation of the master regulator FOXM1 in cancer. Cell Commun Signal 16: 57, 2018

45. Kong X, Li L, Li Z, Le X, Huang C, Jia Z, Cui J, Huang S, Wang L and Xie K: Dysregulated expression of FOXM1 isoforms drives progression of pancreatic cancer. Cancer Res 73: 3987-3996, 2013.

46. Cheng XH, Black M, Ustiyan V, Le T, Fulford L, Sridharan A, Medvedovic M, Kalinichenko VV, Whitsett JA and Kalin TV: SPDEF inhibits prostate carcinogenesis by disrupting a positive feedback loop in regulation of the Foxm1 oncogene. PLoS Genet 10: e1004656, 2014.

47. Korver W, Roose J and Clevers $\mathrm{H}$ : The winged-helix transcription factor Trident is expressed in cycling cells. Nucleic Acids Res 25: 1715-1719, 1997. 
48. Sanders DA, Gormally MV, Marsico G, Beraldi D, Tannahill D and Balasubramanian S: FOXM1 binds directly to non-consensus sequences in the human genome. Genome Biol 16: 130, 2015.

49. Tabatabaei-Dakhili SA, Aguayo-Ortiz R, Domínguez L and Velázquez-Martínez CA: Untying the knot of transcription factor druggability: Molecular modeling study of FOXM1 inhibitors. J Mol Graph Model 80: 197-210, 2018.

50. Van Der Spoel D, Lindahl E, Hess B, Groenhof G, Mark AE and Berendsen HJ: GROMACS: Fast, flexible, and free. J Comput Chem 26: 1701-1718, 2005.

51. Tassi RA, Todeschini P, Siegel ER, Calza S, Cappella P, Ardighieri L, Cadei M, Bugatti M, Romani C, Bandiera E, et al: FOXM1 expression is significantly associated with chemotherapy resistance and adverse prognosis in non-serous epithelial ovarian cancer patients. J Exp Clin Cancer Res 36: 63, 2017.

52. Ye H, Kelly TF, Samadani U, Lim L, Rubio S, Overdier DG, Roebuck KA and Costa RH: Hepatocyte nuclear factor 3/fork head homolog 11 is expressed in proliferating epithelial and mesenchymal cells of embryonic and adult tissues. Mol Cell Biol 17: 1626-1641, 1997.

53. Yao KM, Sha M, Lu Z and Wong GG: Molecular analysis of a novel winged helix protein, WIN. Expression pattern, DNA binding property, and alternative splicing within the DNA binding domain. J Biol Chem 272: 19827-19836, 1997.
54. Gormally M, Marsico G, Rai G, Lowe C, Thomas C, Maloney D, Michael S, Matak-Vincovic D, Jadhav A, Simeonov A and Balasubramanian S: Abstract 3088: Transcription factor as target: Novel small molecule inhibits FOXM1 DNA binding and oncogenic gene products. Cancer Res 76: 3088-3088, 2016.

55. Gormally MV, Dexheimer TS, Marsico G, Sanders DA, Lowe C, Matak-Vinković D, Michael S, Jadhav A, Rai G, Maloney DJ, et al: Suppression of the FOXM1 transcriptional programme via novel small molecule inhibition. Nat Commun 5: $5165,2014$.

56. Marsico G and Gormally MV: Small molecule inhibition of FOXM1: How to bring a novel compound into genomic context. Genom Data 3: 19-23, 2015.

57. Marklund EG, Mahmutovic A, Berg OG, Hammar P, van der Spoel D, Fange D and Elf J: Transcription-factor binding and sliding on DNA studied using micro- and macroscopic models. Proc Natl Acad Sci USA 110: 19796-19801, 2013.

58. Zhang X, Cheng L, Minn K, Madan R, Godwin AK, Shridhar V and Chien J: Targeting of mutant p53-induced FoxM1 with thiostrepton induces cytotoxicity and enhances carboplatin sensitivity in cancer cells. Oncotarget 5: 11365-11380, 2014.

This work is licensed under a Creative Commons Attribution-NonCommercial-NoDerivatives 4.0 International (CC BY-NC-ND 4.0) License. 